International Journal of Geometric Methods in Modern Physics Vol. 5, No. 6 (2008) 963-971

(C) World Scientific Publishing Company

\title{
NONSINGULAR COMPLEX INSTANTONS ON EUCLIDEAN SPACETIME
}

\author{
LLOHANN DALLAGNOL and MARCOS JARDIM \\ Instituto de Matemática, Estatıstica e Computação Cientıfica \\ Universidade Federal de Campinas - UNICAMP \\ Caixa Postal 6065, 13083-859, Campinas, SP, Brazil
}

Received 4 March 2008

Accepted 17 June 2008

\begin{abstract}
Building on a variation of 't Hooft's harmonic function ansatz for $S U(2)$ instantons on $\mathbb{R}^{4}$, we provide new explicit nonsingular solutions of the Yang-Mills anti-self-duality equations on Euclidean spacetime with gauge group $S L(2, \mathbb{C})$ and $S L(3, \mathbb{R})$.
\end{abstract}

Keywords: Instantons; periodic instantons.

\section{Introduction}

Yang-Mills theory has been a rich source of profound mathematical results in the past three decades. It has found applications in a wide variety of research areas, such as differential topology and algebraic geometry [5], representation theory [14], and in the theory of integrable systems [12], to name a few. Usually, one considers the Yang-Mills anti-self-duality (ASD) equations (and its various dimensional reductions) for connections taking values on the Lie algebra of a compact real Lie group. So far, Yang-Mills instantons for complex Lie groups and for noncompact real Lie groups have received little attention, see for instance $[3,4,15,16]$. However, complex gauge theory has appeared in several recent papers, e.g. $[6,9,13]$; in particular, there exists an extensive literature on flat connections with values on complex and real noncompact Lie groups, see for instance [1] and the references therein. Furthermore, no general theory has been developed and the proper physical interpretation of such objects is yet to be understood (see [6] for a related discussion).

In this paper we provide explicit nonsingular solutions of the Yang-Mills antiself-duality equations on Euclidean spacetime with gauge group $S L(2, \mathbb{C})$ and $S L(3, \mathbb{R})$. As it is well-known (see $[8,17]$ ), every complex instanton over $\mathbb{C}^{4}$, regarded as the complexified spacetime, must be singular along a six dimensional submanifold. Thus if we restrict a generic singular instanton on $\mathbb{C}^{4}$ to the real Euclidean (or Minkowiski) spacetime $\mathbb{R}^{4} \subset \mathbb{C}^{4}$ we can expect to end up with a complex instanton 
which is singular along a 2-dimensional subvariety of $\mathbb{R}^{4}$. So the existence of smooth anti-self-dual $S L(n, \mathbb{C})$-connections on $\mathbb{R}^{4}$ is not an obvious fact.

Our starting point is a complex version of the harmonic function ansatz due to 't Hooft and outlined in [11]. We show that to each holomorphic function $f: \mathbb{C}^{2} \rightarrow \mathbb{C}$ one can associate a smooth, anti-self-dual $S L(2, \mathbb{C})$-connection on $\mathbb{R}^{4}$ of zero action density which is not pure gauge. Motivated by the extensive literature on the explicit construction of $S U(2)$-calorons (see $[2,10]$ and the references therein), and the partial results so far obtained on the construction of explicit doubly-periodic $S U(2)$ instantons [7], we also give explicit examples of complex calorons and complex doubly-periodic instantons with the same properties.

Complex instantons with zero action density have been found before, cf. $[3,4,15]$; they were called voidons in [4]. The vanishing of the action density may actually be an advantage from the point of view of the semi-classical approximation, see the discussion in [3]. More recently, $S O(2,1)$-instantons with zero action density were also found in [13], and an interesting connection with the theory of integrable systems was uncovered. Here, integrability once again appears, in the form of the Cauchy-Riemann equations.

\section{Complex 't Hooft Ansatz}

We begin by recalling the harmonic function ansatz due to 't Hooft and presented by Jackiw, Nohl and Rebbi in [11], slightly adapted to fit our purposes. Let $x_{1}, x_{2}, x_{3}, x_{4}$ be coordinates of the Euclidean spacetime $\mathbb{R}^{4}$. Let $\left\{E_{1}, E_{2}, E_{3}\right\}$ be a basis for the Lie algebra $\mathfrak{s u}(2)$ satisfying the commutation relations $\left[E_{i}, E_{j}\right]=\epsilon^{i j k} E_{k}$; for instance, one may consider $E_{j}=\frac{1}{2 i} \sigma_{j}$, where $\sigma_{j}$ are the Pauli matrices. Defining

$$
\bar{\sigma}_{i j}=\left[E_{i}, E_{j}\right] ; \quad \bar{\sigma}_{i 4}=E_{i}
$$

it is easy to check that

$$
\bar{\sigma}_{i j}=\frac{1}{2} \sum_{k, l} \varepsilon_{i j k l} \bar{\sigma}_{k l} \equiv * \bar{\sigma}_{i j} .
$$

Now consider the $S U(2)$-connection given by the expression $A=\sum_{\mu=1}^{4} A_{\mu} d x_{\mu}$ with

$$
A_{\mu}=\sum_{\nu=1}^{4} \rho_{\nu} \bar{\sigma}_{\mu \nu}
$$

where $\rho_{\nu}: \mathbb{R}^{4} \rightarrow \mathbb{R}$ are differentiable functions $(\nu=1, \ldots, 4)$. It is not difficult to see that this connection is anti-self-dual provided the following two conditions are satisfied:

$$
\begin{gathered}
f_{\mu \nu} \equiv \partial_{\mu} \rho_{\nu}-\partial_{\nu} \rho_{\mu}=* f_{\mu \nu} \\
\sum_{\nu=1}^{4}\left(\partial_{\nu} \rho_{\nu}-\rho_{\nu}^{2}\right)=0
\end{gathered}
$$


If $\rho_{\nu}=-\log f \nu$ for some non-vanishing function $f: \mathbb{R}^{4} \rightarrow \mathbb{R}$, we end up with the simpler expression,

$$
\frac{1}{f} \nabla^{2} f=0
$$

In summary, given a non-vanishing harmonic function $f: \mathbb{R}^{4} \rightarrow \mathbb{R}$, the $S U(2)$ connection on $\mathbb{R}^{4}$ given by the expression $A=\sum_{\mu=1}^{4} A_{\mu} d x_{\mu}$ with

$$
A_{\mu}=-\sum_{\nu=0}^{4} \bar{\sigma}_{\mu \nu} \frac{\partial}{\partial x_{\nu}} \ln (f(x))
$$

has anti-self-dual curvature. For instance, if one considers

$$
f(x)=1+\sum_{i=1}^{k} \frac{\lambda_{i}^{2}}{\left(x-y_{i}\right)^{2}},
$$

where $\lambda_{i} \in \mathbb{R}, y_{i} \in \mathbb{R}^{4}$ and $k \geq 0$ is a positive integer, one gets, after an appropriate gauge transformation, $k$ 'basic instantons' with centers $y_{i}$ and scales $\lambda_{i}$.

The simplest way of constructing complex instantons would be to consider $f: \mathbb{R}^{4} \rightarrow \mathbb{C}$, i.e. allowing the superpotential to assume complex values. In other words, consider a pair of nonvanishing harmonic functions $u, v: \mathbb{R}^{4} \rightarrow \mathbb{R}$ and take $f=u+i v$ into formula (7). This will lead to an $S U(2)$-connection with complexvalued coefficients, which may be regarded as an $S L(2, \mathbb{C})$-connection. Notice that the connection produced in this way may, in principle, have arbitrary topological charge.

Now we assume the connection (3) has complex-valued coefficients, i.e. $\rho_{\nu}$ : $\mathbb{R}^{4} \rightarrow \mathbb{C}$. Condition (5) is clearly satisfied if we take $i \rho_{1}=\rho_{3}$ and $i \rho_{2}=\rho_{4}$. It then follows that $\sum \rho_{\nu}^{2}=0$, hence (5) reduces to

$$
\sum_{\nu=1}^{4} \partial_{\nu} \rho_{\nu}=0 .
$$

It is interesting to remark that if we introduce the matrix $J_{\nu}^{\mu}=\partial_{\nu} \rho_{\mu}$, then conditions (4) and (9) become $J=J^{\mathrm{t}}$ and $\operatorname{Tr} J=0$, respectively. Without loss of generality, we can just focus our attention on the symmetry condition. A simple computation is sufficient to conclude that this condition is equivalent to the following equations $(j=1,2)$ :

$$
\begin{aligned}
i \partial_{1} \rho_{j} & =\partial_{3} \rho_{j}, \\
i \partial_{2} \rho_{j} & =\partial_{4} \rho_{j}, \\
\partial_{2} \rho_{1} & =\partial_{1} \rho_{2} .
\end{aligned}
$$

Turning to complex coordinates $u=x_{1}+i x_{3}, v=x_{2}+i x_{4}$, it is not difficult to see that (10) and (11) are exactly the Cauchy-Riemann equations for $\rho_{1}$ and $\rho_{2}$. 
With all these assumptions, the coefficients of the connection $A=A_{\nu} d x^{\nu}$ are given by

$$
\begin{aligned}
& A_{1}=i \rho_{2} E_{1}-i \rho_{1} E_{2}+\rho_{2} E_{3}, \\
& A_{2}=i \rho_{1} E_{1}+i \rho_{2} E_{2}-\rho_{1} E_{3}, \\
& A_{3}=i A_{1}, \quad A_{4}=i A_{2},
\end{aligned}
$$

with curvature components

$$
\begin{aligned}
F_{12}= & i\left(\partial_{1} \rho_{1}-\partial_{2} \rho_{2}+\rho_{1}^{2}-\rho_{2}^{2}\right) E_{1}+2 i\left(\partial_{2} \rho_{1}+\rho_{1} \rho_{2}\right) E_{2} \\
& -\left(\partial_{1} \rho_{1}+\partial_{2} \rho_{2}+\rho_{1}^{2} \rho_{2}^{2}\right) E_{3}, \\
F_{23}= & -i F_{12}, \\
F_{13}= & 0 .
\end{aligned}
$$

Moreover, the ASD condition holds, i.e.:

$$
F_{12}=-F_{34}, \quad F_{23}=-F_{14} \quad \text { and } \quad F_{13}=F_{24} .
$$

As usual, we define the topological charge of an instanton by the expression (also considered in [4]):

$$
W=-\frac{1}{8 \pi^{2}} \int_{\mathbb{R}^{4}} \operatorname{Tr}(F \wedge F) .
$$

We remark that the above expression is $S L(2, \mathbb{C})$ gauge invariant. However, the bilinear form $(A, B)=\operatorname{Tr}(A B)$ is not positive definite in $\mathfrak{s l}(2, \mathbb{C})$, so $W$ may vanish even though $F$ is not pure gauge.

It is easy to check that if $A$ is anti-self-dual, then $F \wedge F=F_{12}^{2}+F_{13}^{2}+F_{23}^{2}$; in particular, our connections satisfy

$$
F \wedge F=F_{12}^{2}+\left(-i F_{12}\right)^{2}=0 .
$$

In other words, all connections constructed in this section have zero energy density.

\section{Instantons with Holomorphic Conditions}

Let $u=x_{1}+i x_{3}, v=x_{2}+i x_{4}$ be complex coordinates, so that

$$
\begin{array}{r}
d u=\frac{1}{2}\left(d x^{1}+i d x^{3}\right), \quad d v=\frac{1}{2}\left(d x^{2}+i d x^{4}\right), \\
\partial_{u}=\partial_{1}-i \partial_{3}, \quad \partial_{v}=\partial_{2}-i \partial_{4}, \\
d \bar{u}=\frac{1}{2}\left(d x^{1}-i d x^{3}\right), \quad d \bar{v}=\frac{1}{2}\left(d x^{2}-i d x^{4}\right), \\
\partial_{\bar{u}}=\partial_{1}+i \partial_{3}, \quad \partial_{\bar{v}}=\partial_{2}+i \partial_{4} .
\end{array}
$$

One can check that the following equality holds

$$
A=A_{\nu} d x^{\nu}=\left(A_{1}-i A_{3}\right) d u+\left(A_{2}-i A_{4}\right) d v+\left(A_{1}+i A_{3}\right) d \bar{u}+\left(A_{2}+i A_{4}\right) d \bar{v} .
$$


We fix the notation $A_{u}, A_{v}, A_{\bar{u}}, A_{\bar{v}}$ for the corresponding components of $A$ in the complex coordinates above. Using the notation

$$
F_{\mu \nu}=\partial_{\mu} A_{\nu}-\partial_{\nu} A_{\mu}+\left[A_{\nu}, A_{\mu}\right], \quad \mu, \nu=u, v, \bar{u}, \bar{v},
$$

we have the ASD conditions for $A$ expressed as

$$
F_{u \bar{v}}=0, \quad F_{v \bar{u}}=0, \quad F_{u \bar{u}}=F_{v \bar{v}}
$$

while the energy density in the ASD case is given by

$$
\operatorname{Tr}\left(F_{12}^{2}+F_{23}^{2}+F_{13}^{2}\right)=\frac{1}{4} \operatorname{Tr}\left(F_{u v} F_{\bar{u} \bar{v}}-F_{u \bar{u}}^{2}\right) .
$$

Now we suppose that $A_{\bar{u}}=A_{\bar{v}}=0$, hence the ASD conditions (19) simplify to

$$
\partial_{\bar{v}} A_{u}=0, \quad \partial_{\bar{u}} A_{v}=0, \quad \partial_{\bar{u}} A_{u}=\partial_{\bar{v}} A_{v} .
$$

The curvature components of such a connection are then given by

$$
\begin{aligned}
& F_{u v}=\partial_{u} A_{v}-\partial_{v} A_{u}+\left[A_{u}, A_{v}\right], \\
& F_{\bar{u} \bar{v}}=0, \\
& F_{u \bar{u}}=-\partial_{\bar{u}} A_{u},
\end{aligned}
$$

with energy density

$$
\operatorname{Tr}(F \wedge F)=-\frac{1}{4} \operatorname{Tr}\left\{\left(\partial_{\bar{u}} A_{u}\right)^{2}\right\} .
$$

From the condition $A_{\bar{u}}=A_{\bar{v}}=0$ and (21), we have that:

$$
\partial_{\bar{v}} A_{1}=0, \quad \partial_{\bar{u}} A_{2}=0, \quad \partial_{\bar{v}} A_{2}=\partial_{\bar{u}} A_{1}
$$

and

$$
i A_{1}=A_{3}, \quad i A_{2}=A_{4} .
$$

Summing up, we conclude that if $A=A_{\nu} d x^{\nu}$ is a connection satisfying (26) and (27), then $A$ is a ASD. Furthermore, if $\partial_{\bar{u}} A_{1}=0$, then the energy density (hence the topological charge) of $A$ vanishes. One can easily notice from (13) that the complex 't Hooft ansatz obtained in the previous section is a particular case of the holomorphic instanton constructed above. It is also worth noting that, by the weaker conditions exposed in this section, (12) can be neglected without loss of anti-self-duality.

\section{Charge Zero $S L(n, \mathbb{R})$ Instantons for $n \geq 3$}

We now assume a reality condition on $A$ by supposing that $A_{\bar{u}}=\bar{A}_{u}$ and $A_{\bar{v}}=$ $\bar{A}_{v}$, i.e. the matrices $A_{i}$ have real valued coefficients. We further assume that the complex coefficients of $A$ are holomorphic, i.e. $\partial_{\bar{u}} A_{u}=\partial_{\bar{v}} A_{u}=\partial_{\bar{u}} A_{v}=\partial_{\bar{v}} A_{v}=0$; we also have $\partial_{u} A_{\bar{u}}=\partial_{u} A_{\bar{v}}=\partial_{u} A_{\bar{v}}=\partial_{v} A_{\bar{v}}=0$ since the former is the complex 
conjugate of the latter. In this situation, from (19), the ASD condition simplifies to a purely algebraic condition:

$$
\begin{aligned}
& {\left[A_{u}, A_{\bar{u}}\right]=\left[A_{v}, A_{\bar{v}}\right]} \\
& {\left[A_{u}, A_{\bar{v}}\right]=0} \\
& {\left[A_{v}, A_{\bar{u}}\right]=0 .}
\end{aligned}
$$

One can also check that (29) is equivalent to (30).

Now we suppose

$$
A_{u}=\rho(x) M, \quad A_{v}=\mu(x) M,
$$

for $\rho, \mu$ holomorphic functions. Equations (28) and (29) became

$$
\begin{aligned}
\rho \mu[M, \bar{M}] & =0 \\
|\rho|^{2}[M, \bar{M}] & =|\mu|^{2}[M, \bar{M}] .
\end{aligned}
$$

As one can see the interesting case is $[M, \bar{M}]=0$. Setting $M=a+i b$ with $a, b$ real matrices, then $[M, \bar{M}]=0$ iff $[a, b]=0$. Unfortunately, if $a, b \in \mathfrak{s l}(2, \mathbb{R})$ then $a$ and $b$ are multiple of each other, and the corresponding instanton would turn out to be abelian. However, for $n \geq 3$, there exist pairs of traceless commuting matrices that are not multiple of one another, see the examples below.

The energy density of this connection will be

$$
\operatorname{Tr}(F \wedge F)=-\left(\left|\partial_{v} \rho-\partial_{u} \mu\right|^{2} \operatorname{Tr} M \bar{M}\right) d u \wedge d v \wedge d \bar{u} \wedge d \bar{v} .
$$

Concluding that the charge will be zero if the right-hand side vanishes, i.e. when either $\partial_{v} \rho=\partial_{u} \mu$ or $\operatorname{Tr} M \bar{M}=\operatorname{Tr}\left(a^{2}+b^{2}\right)=0$.

\section{Explicit Examples}

A good way for constructing examples is to take some $\rho: \mathbb{C}^{2} \rightarrow \mathbb{C}$ holomorphic in $z_{1}, z_{2}$ and make $\rho_{j}=\frac{\partial \rho}{\partial z_{j}}$. This is enough for conditions (10)-(12). Here we let $E_{j}=\frac{1}{2 i} \sigma_{j}$ where $\sigma_{j}$ are the Pauli matrices. We use in this section the notation $z_{1}=x_{1}+i x_{3}, z_{2}=x_{2}+i x_{4}$.

Example 1. Taking $\rho=z_{1} z_{2}$ we have $\rho_{1}=z_{2}$ and $\rho_{2}=z_{1}$. Remembering that the curvature is completely defined with its $F_{12}$ component as seen in (14) and by (13) we have

$$
\begin{gathered}
A_{1}=-i A_{3}=\frac{1}{2}\left(\begin{array}{cc}
-i z_{1} & z_{1}+i z_{2} \\
z_{1}-i z_{2} & i z_{1}
\end{array}\right), \\
A_{2}=-i A_{4}=\frac{1}{2}\left(\begin{array}{cc}
i z_{2} & z_{2}-i z_{1} \\
z_{2}+i z_{1} & -i z_{2}
\end{array}\right), \\
F_{12}=i\left(z_{2}^{2}-z_{1}^{2}\right) E_{1}+2 i\left(1+z_{1} z_{2}\right) E_{2}-\left(z_{2}^{2}+z_{1}^{2}\right) E_{3} .
\end{gathered}
$$


This is a nonsingular charge zero instanton with nonvanishing curvature. The vanishing energy density (16) and the nonzero curvature contrasts with the $S U(2)$ case where the energy density vanishes only when the curvature vanishes.

Example 2 (Complex Caloron). Setting $\rho=z_{1} e^{z_{2}}$, we have $\rho_{1}=e^{z_{2}}$ and $\rho_{2}=z_{1} e^{z_{2}}$. By (13) and (14) the connection and curvature will be

$$
\begin{gathered}
A_{1}=-i A_{3}=\frac{e^{z_{2}}}{2}\left(\begin{array}{cc}
-i z_{1} & z_{1}+i \\
z_{1}-i & i z_{1}
\end{array}\right), \\
A_{2}=-i A_{4}=\frac{e^{z_{2}}}{2}\left(\begin{array}{cc}
i & 1-i z_{1} \\
1+i z_{1} & -i
\end{array}\right), \\
F_{12}=i e^{z_{2}}\left(e^{z_{2}}-z_{1}-z_{1}^{2} e^{z_{2}}\right) E_{1}+2 i e^{z_{2}}\left(1+z_{1} e^{z_{2}}\right) E_{2}-z_{1} e^{z_{2}}\left(1+z_{1} e^{3 z_{2}}\right) E_{3} .
\end{gathered}
$$

This solution is nonsingular and periodic in the $x_{4}$ direction, i.e. it is a complex caloron. It also has zero energy density.

Example 3 (Complex doubly-periodic instanton). Taking $\rho=e^{z_{1}} e^{z_{2}}$, we have $\rho_{1}=e^{z_{1}} e^{z_{2}}$ and $\rho_{2}=e^{z_{1}} e^{z_{2}}$. Again by (13) and (14) we arrive at

$$
\begin{aligned}
& A_{1}=-i A_{3}=\frac{e^{z_{1}+z_{2}}}{2}\left(\begin{array}{cc}
-i & 1+i \\
1-i & i
\end{array}\right), \\
& A_{2}=-i A_{4}=\frac{e^{z_{1}+z_{2}}}{2}\left(\begin{array}{cc}
i & 1-i \\
1+i & -i
\end{array}\right), \\
& F_{12}=2 i\left(e^{z_{1}} e^{z_{2}}+e^{2 z_{1}} e^{2 z_{2}}\right)\left(E_{2}-E_{3}\right) .
\end{aligned}
$$

This solution is nonsingular and periodic in the $x_{2}$ and $x_{4}$ directions, so it is a complex doubly-periodic instanton. It also has zero energy density. As far as we know, this is the first explicit example of a doubly-periodic instanton.

Now we give an example of complex solution that is not from the above complex 't Hooft ansatz.

Example 4. Setting $\rho_{1}=z_{1} e^{z_{2}}$ and $\rho_{2}=e^{z_{1}} z_{2}$ in the construction of Sec. 3, one obtains:

$$
\begin{aligned}
A_{1} & =\frac{1}{2}\left(\begin{array}{cc}
-i e^{z_{1}} z_{2} & e^{z_{1}} z_{2}+i z_{1} e^{z_{2}} \\
e^{z_{1}} z_{2}-i z_{1} e^{z_{2}} & i e^{z_{1}} z_{2}
\end{array}\right) \\
A_{2} & =\frac{1}{2}\left(\begin{array}{cc}
i z_{1} e^{z_{2}} & z_{1} e^{z_{2}}-i e^{z_{1}} z_{2} \\
z_{1} e^{z_{2}}+i e^{z_{1}} z_{2} & -i z_{1} e^{z_{2}}
\end{array}\right) .
\end{aligned}
$$

Therefore,

$$
\begin{aligned}
F_{12}= & i\left(e^{z_{2}}-e^{z_{1}}+z_{1}^{2} e^{2 z_{2}}-z_{2}^{2} e^{2 z_{1}}\right) E_{1}+i\left(z_{2} e^{z_{1}}+z_{1} e^{z_{2}}+2 z_{1}^{2} z_{2}^{2} e^{2 z_{1}} e^{2 z_{2}}\right) E_{2} \\
& -\left(e^{z_{1}}+e^{z_{2}}+z_{1}^{2} z_{2}^{2} e^{2\left(z_{1}+z_{2}\right)}\right) E_{3} .
\end{aligned}
$$

Note that Eq. (13) do not hold, showing that this example does not arise from the complex 't Hooft ansatz. 
Finally, we conclude with an explicit example of a $S L(3, \mathbb{R})$ instanton with nonzero curvature but zero action density.

Example $5\left(\boldsymbol{S L}(\mathbf{3}, \mathbb{R})\right.$ instanton). We consider in Eq. (31) $\rho=2 e^{z_{2}}, \mu=2 z_{1}$ with $M=a+i b$,

$$
a=\left(\begin{array}{lll}
0 & 1 & 0 \\
0 & 0 & 0 \\
0 & 0 & 0
\end{array}\right), \quad b=\left(\begin{array}{lll}
0 & 0 & 1 \\
0 & 0 & 0 \\
0 & 0 & 0
\end{array}\right)
$$

It follows that

$$
\begin{aligned}
& A_{1}=e^{x_{2}}\left(\cos x_{4} a-\sin x_{4} b\right), \\
& A_{2}=x_{1} a-x_{3} b, \\
& A_{3}=e^{x_{2}}\left(\sin x_{4} a+\cos x_{4} b\right), \\
& A_{4}=x_{3} a+x_{1} b,
\end{aligned}
$$

with vanishing energy density and the $F_{u v}$ component of the complex curvature is

$$
F_{u v}=F_{12}-F_{34}+i\left(F_{23}-F_{14}\right)=4\left(1-2 e^{z_{2}}\right) M .
$$

So the curvature in real coordinates cannot be zero, concluding that it is not a pure gauge connection and contrasting again with the $S U(n)$ case.

\section{Conclusion}

In this paper we presented two ways of constructing explicit, nonsingular complex instantons on the Euclidean $\mathbb{R}^{4}$ with zero action density. Further examples could be obtained by replacing the conditions $A_{\bar{u}}=A_{\bar{v}}=0$ and $A_{\bar{u}}=\bar{A}_{u}, A_{\bar{v}}=\bar{A}_{v}$, imposed respectively in Sec. 3 and in Sec. 4, by another simplifying assumption, showing that our method is not completely exhausted. We hope that these examples will be useful as motivation for the development of a general deformation theory of complex instantons, and lead to better understanding of its physical interpretation and of the structure of its moduli spaces.

\section{Acknowledgments}

LD's research was supported by the FAPESP grant number 2005/04489-8. MJ is partially supported by the CNPq grant number 305464/2007-8. This project was also supported by the FAPESP grant number 2005/04558-0. We thank Ricardo A. Mosna for many useful discussions.

\section{References}

[1] S. B. Bradlow, O. Garcia-Prada and P. B. Gothen, Maximal surface group representations in isometry groups of classical Hermitian symmetric spaces, Geom. Dedicata 122 (2006) 185-213. 
[2] F. Bruckmann, D. Nogradi and P. van Baal, Higher charge calorons with non-trivial holonomy, Nuc. Phys. B 698 (2004) 233-254.

[3] A. D. Burns, Complex string solutions of the self-dual Yang-Mills equations, J. Phys. A 17 (1984) 689-707.

[4] L. Dolan, Complex solutions of Yang-Mills, Phys. Lett. B 76 (1978) 105.

[5] S. K. Donaldson and P. B. Kronheimer, The Geometry of Four-manifolds (Claredon Press, Oxford, 1990).

[6] G. Etesi, Gravitational interpretation of the Hitchin equations, J. Geom. Phys. 57 (2007) 1778-1788.

[7] C. Ford and J. M. Pawlowski, Doubly periodic instantons and their constituents, Phys. Rev D 69 (2004) 065006.

[8] I. B. Frenkel and M. Jardim, Complex ADHM equations and sheaves on $\frac{\partial}{\partial x_{3}}$, J. Algebra 319 (2008) 2913-2937.

[9] S. Gukov and H. Murakami, SL $(2, \mathrm{C})$ Chern-Simons theory and the asymptotic behavior of the colored Jones polynomial, preprint math/0608324.

[10] D. Harland, Large scale and large period limits of symmetric calorons, J. Math. Phys. 48 (2007) 082905.

[11] R. Jackiw, C. Nohl and C. Rebbi, Conformal properties of pseudo particle configurations, Phys. Rev. D 15 (1977) 1642-1646.

[12] L. J. Mason and N. M. J. Woodhouse, Integrability, Self-duality, and Twistor Theory (Clarendon Press, Oxford, 1996).

[13] R. A. Mosna and M. Jardim, Nonsingular solutions of Hitchin's equations for noncompact gauge groups, Nonlinearity 20 (2007) 1893-1906.

[14] H. Nakajima, Instantons on ALE spaces, quiver varieties, and Kac-Moody algebras, Duke Math. J. 76 (1994) 365-416.

[15] C. H. Oh and R. Teh, Linearly superposable and finite-action classical Yang-Mills fields, J. Math. Phys. 22 (1981) 585-589.

[16] C. Rebbi, Self-dual Yang-Mills fields in Minkowiski space-time, Phys. Rev. D 17 (1978) 483-485.

[17] R. Ward and R. O. Wells, Twistor Geometry and Field Theory (Cambridge University Press, Cambridge, 1990). 